\title{
Photobiostimulation reverses allodynia and peripheral nerve damage in streptozotocin-induced type 1 diabetes
}

\author{
Igor Rafael Correia Rocha ${ }^{1}$ - Adriano Polican Ciena ${ }^{2}$ - Alyne Santana Rosa ${ }^{1}$. \\ Daniel Oliveira Martins ${ }^{1} \cdot$ Marucia Chacur $^{1}$
}

Received: 1 November 2016 / Accepted: 26 December 2016 / Published online: 30 January 2017

(C) Springer-Verlag London 2017

\begin{abstract}
For better evaluation of the efficacy of low-level laser therapy in treating painful diabetic neuropathy and in protecting nerve fiber damage, we conducted a study with type 1 diabetic rats induced by streptozotocin. It is well known that diabetic peripheral neuropathy is the leading cause of pain in those individuals who suffer from diabetes. Despite the efficacy of insulin in controlling glucose level in blood, there is no effective treatment to prevent or reverse neuropathic damage for total pain relief.Male Wistar rats were divided into saline, vehicle, and treatment groups. A single intraperitoneal (i.p.) injection of streptozotocin (STZ) $(85 \mathrm{mg} / \mathrm{kg}$ ) was administered for the induction of diabetes. The von Frey filaments were used to assess nociceptive thresholds (allodynia). Behavioral measurements were accessed 14, 28, 48, and 56 days after STZ administration. Rats were irradiated with GaAs Laser (Gallium Arsenide, Laserpulse, Ibramed Brazil)
\end{abstract}

Marucia Chacur

chacurm@icb.usp.br

Igor Rafael Correia Rocha

ircrocha@usp.br

Adriano Polican Ciena

apciena@rc.unesp.br

Alyne Santana Rosa

alynesrosa@usp.br

Daniel Oliveira Martins

martinsd@usp.br

1 Department of Anatomy, Biomedical Sciences Institute, University of São Paulo, Av. Prof. Lineu Prestes, 2415, 05508-900 São Paulo, Brazil

2 Institute of Biosciences, University Estadual Paulista Júlio de Mesquita Filho, Rio Claro, 13506900 São Paulo, Brazil emitting a wavelength of $904 \mathrm{~nm}$, an output power of 45 mWpk, beam spot size at target $0.13 \mathrm{~cm}^{2}$, a frequency of $9500 \mathrm{~Hz}$, a pulse time $60 \mathrm{~ns}$, and an energy density of $6,23 \mathrm{~J} / \mathrm{cm}^{2}$. The application of four sessions of low-level laser therapy was sufficient to reverse allodynia and protect peripheral nerve damage in diabetic rats. The results of this study indicate that low-level laser therapy is feasible to treat painful diabetic condition in rats using this protocol. Although its efficacy in reversing painful stimuli and protecting nerve fibers from damage was demonstrated, this treatment protocol must be further evaluated in biochemical levels to confirm its biological effects.

Keywords Diabetes mellitus $\cdot$ Sciatic nerve $\cdot$ Nociception $\cdot$ Myelin sheath $\cdot$ Rat $\cdot$ Streptozotocin

\section{Abbreviations \\ i.p. Intraperitoneal \\ STZ Streptozotocin}

\section{Introduction}

Diabetes is a chronic condition that occurs when the body cannot produce or use enough insulin, and it is diagnosed by increased levels of glucose in the blood. Insulin is a hormone produced by $\beta$ cells in the pancreas; it is required to transport glucose from the bloodstream into the cells where it is used as energy. The lack or ineffectiveness of insulin in a person with diabetes results in high glucose concentration in the blood. Over time, the resulting high levels of glucose in the blood (hyperglycemia) cause damage to several tissues in the body, especially in the nervous system, leading to the development of disabilities and life-threatening health complications [1]. 
Diabetic peripheral neuropathy (DPN) is a group of heterogeneous disorders caused by neuronal dysfunction, and it affects approximately $50 \%$ of patients with diabetes mellitus. These disorders show different clinical courses, distributions, fiber involvement (large and small), and pathophysiologies [2]. DPN is thought to occur as a result of two situations: (1) Hyperglycemia-induced damage to nerve cells; (2) Neuronal ischemia caused by the decrease in neurovascular flow due to hyperglycemia [3]. Both axonal loss and sensory nerve fiber dysfunction [4] occur in diabetes mellitus but the exact pathomechanism underlying nerve damage in diabetes remains unknown.

Diabetic peripheral neuropathy is a complication of diabetes that comprises functional and structural changes in peripheral nerves, such as a reduction of velocity in nerve conduction, axonal degeneration, paranodal demyelination, and loss of myelinated and unmyelinated fibers. Some of the morphological alterations in peripheral nerve fibers associated with hyperglycemia are also seen in rat models of STZ-induced diabetic neuropathy [5].

Alterations in myelin damage include invaginations in the axoplasm (infoldings) and myelin evaginations in the Schwann cell cytoplasm (outfoldings). These changes also include myelin compaction such as abnormal wide incisures and aberrant separation of myelin lamellae, similar to those seen in aged rodents [6]. The predominant myelin abnormalities found in STZ-treated rats are myelin infoldings [7].

The myelin sheath is a multilayered membrane produced in the peripheral nervous system by differentiation of the plasma membrane of Schwann cells. The main role of this membrane is to allow efficient transmission of nerve impulses along the axons that it surrounds [5].

Over time, people with diabetes mellitus may or may not develop symptoms such as severe pain, tingling, numbness, and loss of sensation in the hands and feet [8]. Painful diabetic neuropathy is the most common manifestation of diabetes [9], and it appears as a burning, excruciating, and stabbing intractable type of pain [10].

Treatment for painful diabetic neuropathy is based on intensive glycemic control and symptomatic pain management [2]. This includes antidepressants like tricyclic antidepressants (TCAs), selective norepinephrine reuptake inhibitors (SNRIs), anticonvulsants including pregabalin, gabapentin and lamotrigine, and also topical agents such as capsaicin [10].

Despite all these pharmacological agents available for pain relief, their efficacy is limited due to several side effects. There are no therapeutic approaches available for preventing peripheral nerve damage that reverse degeneration or that prevent peripheral unmyelinated nerve fibers loss in patients with diabetes mellitus. The use of alternative approaches for improving pain, preventing peripheral nerve fibers loss, and restoring the quality of life for those suffering from DPN is strongly recommended in all fields of diabetic research.

One of these alternatives is the use of low-level laser therapy, first described in Europe and Russia in the 1960s [11]. Low-level laser therapy is well known for treating chronic pain by increasing nociceptive threshold after its application [12] although its mechanisms of action are not well understood.

The mechanisms by which low-level laser therapy improve neuropathic pain are possibly related to the increase in mitochondrial ATP production [13] and in the release of endorphins [14] as well as a higher release in local antiinflammatory cytokines, such as interleukin-10 [15].

In the present study, we observed myelin sheath structural alterations in a rat model of streptozotocin-induced type 1 diabetes and also a possible correlation between these morphological alterations and behavioral changes.

\section{Methods and materials}

\section{Animals}

Male Wistar rats, weighing between 200 and $220 \mathrm{~g}$ ( 2 months old), were used in all experiments. They were housed in cages (five per cage) and maintained on a 12:12-h light/dark cycle. The rats were adapted to the experimental environment $15 \mathrm{~min}$ prior to the experiments. All animals were tested during the light cycle at the same time of the day (9:00 am-14:00 pm). Due to polyuria, animal bedding was changed twice a day, early in the morning at 8 am and in the night at $8 \mathrm{pm}$. All procedures were approved by the Institutional Animal Care Committee of the University of São Paulo (protocol number 123/2015) and performed in accordance with the guidelines for the ethical use of conscious animals in pain study published by the international association for the study of pain (IASP). Efforts were made to minimize the number of animals used and their suffering [16].

\section{Methods}

\section{Induction of diabetes mellitus}

Diabetes was induced by a single dose $(85 \mathrm{mg} / \mathrm{kg})$ of streptozotocin (STZ, Sigma-Alderich, St. Louis, MO, USA) diluted in saline $0,9 \%$ and administered via intraperitoneal. Blood glucose levels (glycemic control) were assessed from the second day until the end of the experiment using an ULTRAMINI ${ }^{\circledR}$ | ONETOUCH ${ }^{\circledR}$ blood glucose monitoring system (Table 2). A single administration of streptozotocin induced insulin-dependent diabetes mellitus within 24-48 h by destruction of pancreatic islet cells [17]. Plasma glucose levels higher than $300 \mathrm{mg} / \mathrm{dl}$ were considered indicative of 
diabetes [18]. Normal control rats received the same volume of saline $0,9 \%$ (streptozotocin vehicle).

\section{Transmission electron microscopy}

The animals were intraperitoneally anesthetized with urethane ( $3 \mathrm{~g} / \mathrm{kg}$ ) and perfused with a modified Karnovsky fixative solution (containing $2.5 \%$ glutaraldehyde and $2 \%$ paraformaldehyde in $0.1 \mathrm{M}$ sodium phosphate buffer, $\mathrm{pH}$ 7.4) [19]. After dissecting the affected sciatic nerve, samples $(3 \mathrm{~mm})$ were post fixed in a $1 \%$ osmium tetroxide solution at $4{ }^{\circ} \mathrm{C}$ and subsequently immersed in a 5\% uranyl acetate aqueous solution at room temperature. Next, the samples were dehydrated in an increasing alcohol series, immersed in propylene oxide, and embedded in Spur resin. Semi-thin sections were cut with a Reichert Ultra Cut ultra-microtome and stained with a $1 \%$ toluidine blue solution to test the region to be analyzed. Ultrathin $60-\mathrm{nm}$ sections were then cut, collected on 200 "mesh" copper grids (Sigma), and contrasted with a $4 \%$ uranyl acetate solution and $0.4 \%$ lead citrate solution [20]. The grids were observed with a Jeol 1010 transmission electron microscope (Peabody, MA, USA) [21].

\section{Assessment of tactile allodynia}

Rats were transferred to a testing cage with a wire mesh bottom that allowed full access to the paws. Behavioral adaptation was allowed for approximately $15 \mathrm{~min}$, until the cage exploration and major grooming activities had ceased. The area tested was the plantar surface of the animal's hind paw. The von Frey test was used to assess nociceptive thresholds. Briefly, a logarithmic series of 7 calibrated Semmes-Weinstein monofilaments (Von Frey hair test, Stoelting, USA) were applied to the middle of the plantar surface of the right hindpaw, for a maximum of $10 \mathrm{~s}$ to determine the threshold intensity of the stiffness stimulus required to elicit a paw withdrawal response. Log stiffness of the hairs is determined by ranges starting at 4,56 N (3.630 g); 3.61 (0.407 g.); 3.84 (0.692 g.); 4.17 (1.479 g.); 4.93 (8.511 g.); 5.18 (15.136 g.); and 5.46 $(28.840 \mathrm{~g})$. During each testing trial, the series of filaments were presented following an up-down procedure as described and previously validated by Chaplan [22] and the $50 \%$ response threshold was calculated for each rat. The allodynic measurements were accessed on days 14, 28, 48, and 56 after streptozotocin intraperitoneal administration.

\section{Laser therapy}

The laser treatment was performed every other day, totaling 4 sessions, starting on day 45 after the induction of diabetes mellitus type 1. After sterilization, the laser was placed on the skin surface on the right thigh directly above the course of the sciatic nerve. Animals were irradiated with laser GaAs
(Gallium Arsenide, Laserpulse-Laser, Ibramed Brazil) emitting a wavelength of $904 \mathrm{~nm}$, an output power of $45 \mathrm{mWpk}$, a spot area of $0.13 \mathrm{~cm}^{2}$, a frequency of $9500 \mathrm{~Hz}$, a pulse time of $60 \mathrm{~ns}$, and an energy density of $6.23 \mathrm{~J} / \mathrm{cm}^{2}$ (Table 1). Each session included the stimulation of nine points along the sciatic nerve, lasting $18 \mathrm{~s}$ on each point, and 7.29 J of energy per session [23].

\section{Statistical analysis}

Results are presented as the mean \pm SEM. Statistical analyses of data were generated using GraphPAd Prism, version 5 (Graph-Pad Software Inc., San Diego, CA). Statistical comparison of the groups was performed using one-way analysis of variance; differences between means were tested by Bonferroni's multiple comparison test. In all cases, $p<0.05$ was considered statistically significant [24].

\section{Results}

We performed allodynia tests during the time course of 56 days of the development of streptozotocin-induced type 1 diabetes mellitus as described in materials and methods. We also assessed the blood glucose levels during the first 30 days after intraperitoneal administration of streptozotocin to confirm the hyperglycemic state. In addition, we also observed that diabetic rats showed significant loss of body weight and increase

Table 1 Specifications for laser parameters

\begin{tabular}{ll}
\hline $\begin{array}{l}\text { Device information } \\
\text { Manufacturer }\end{array}$ & IBRAMED \\
Model identifier & LASERPULSE Diamond \\
Emitter type & GaAs \\
Irradiation parameters & \\
Wavelength (nm) & $904 \mathrm{~nm}$ \\
Operating mode & Pulsed \\
Frequency $(\mathrm{Hz})$ & 9500 \\
Pulse on duration $(\mathrm{sec})$ & $60 \mathrm{~ns}$ \\
Beam shape & Circular \\
Treatment parameters & \\
Beam spot size at target (cm2) & $0.13 \mathrm{~cm}^{2}$ \\
Exposure duration $(\mathrm{sec})$ & $18 \mathrm{~s} \mathrm{per} \mathrm{point,} \mathrm{162} \mathrm{s} \mathrm{per} \mathrm{session}$ \\
Radiant exposure $(\mathrm{J} / \mathrm{cm} 2)$ & $6.23 \mathrm{~J} / \mathrm{cm}^{2}$ \\
Radiant energy $(\mathrm{J})$ & $0.81 \mathrm{~J}$ \\
Number of points irradiated & 9 points \\
Area irradiated $(\mathrm{cm} 2)$ & $1.17 \mathrm{~cm}^{2}$ \\
Application technique & Skin contact \\
Number and frequency of & 5 sessions, performed every other \\
treatment sessions & day \\
Total radiant energy $(\mathrm{J})$ & $7,291 \mathrm{~J}$ per session; 29,16 J over \\
& all sessions \\
\hline
\end{tabular}


in food and water intake (polyphagia and polydipsia) and also increase in urination (polyuria) (data not shown).

\section{Effect of streptozotocin on blood glucose}

From 24 h until 30 day after streptozotocin administration, diabetic rats $(n=5)$ exhibited significantly increase in blood glucose levels as compared to the levels of the control group (saline) (Fig. 1). In Table 2, we can observe the effect of streptozotocin administration from each animal compared with control animals.

\section{Effect of streptozotocin-induced diabetes mellitus on nociceptive threshold}

When analyzing the allodynic response of the animals with diabetes mellitus, it was possible to observe a significant decrease in nociceptive threshold starting at day 14 after streptozotocin administration and maintained until the end of 56 days when compared with saline animals, used as control (Fig. 2).

\section{Effect of photobiostimulation on nociceptive threshold}

Low-level laser therapy started 45 days after STZ injection. Figure 3 shows a decrease in pain sensitivity initiating after the first session of laser treatment. After four sessions of low level laser therapy on diabetic rats, our data showed significant statistical difference $(p<0.05)$ between diabetic rats before and after the laser application. Furthermore, there were no statistical differences between rats treated with laser and baseline (Fig. 3).

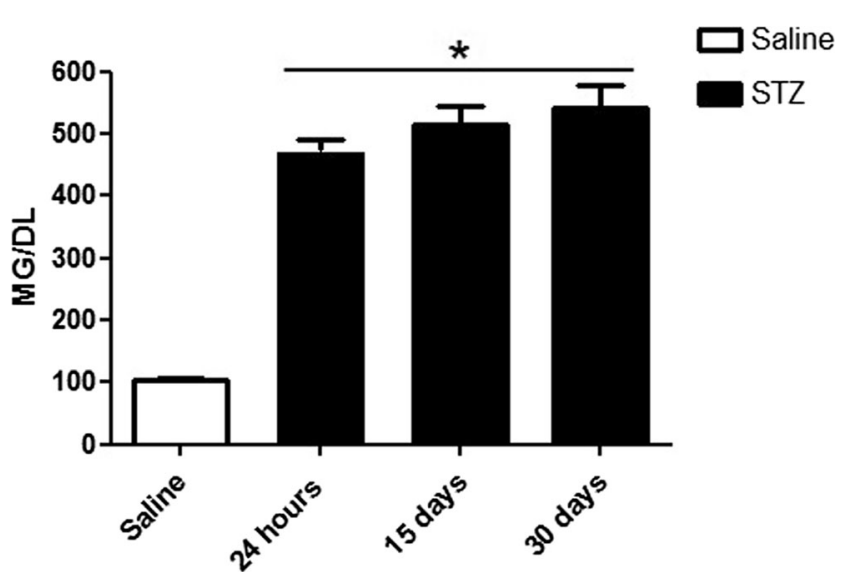

Fig. 1 Blood glucose measurement in saline (control group) and in diabetic rats. Animals were measured with 24 h, 15 days, and 30 days after streptozotocin injection. Results represent the mean $\mathrm{SEM} \pm 5$ animals per group $* P<0.05$ compared with saline
Table 2 Glycemic control were assessed using an ULTRAMINI® | ONETOUCH® blood glucose monitoring system

\begin{tabular}{llll}
\hline $\begin{array}{l}\text { Basal blood } \\
\text { glucose levels } \\
(\mathrm{mg} / \mathrm{d} 1)\end{array}$ & $\begin{array}{l}\text { 24 h after STZ } \\
\text { injection }(\mathrm{mg} / \\
\mathrm{d} 1)\end{array}$ & $\begin{array}{l}\text { 14 days after } \\
\text { STZ injection } \\
(\mathrm{mg} / \mathrm{d} 1)\end{array}$ & $\begin{array}{l}\text { 1 month after } \\
\text { STZ injection } \\
(\mathrm{mg} / \mathrm{d} 1)\end{array}$ \\
\hline 106 & 494 & 509 & 543 \\
115 & 511 & 425 & 400 \\
99 & 453 & 600 & 591 \\
100 & 440 & 515 & 600 \\
95 & 485 & 533 & 573 \\
\hline
\end{tabular}

STZ streptozotocin

Effect of streptozotocin-induced type 1 diabetes mellitus on myelin sheath abnormalities in the sciatic nerve

Electron microscopy analysis showed that sciatic nerve fibers from the control group (saline) exhibited a normal morphology of axons and Schwann cells (Fig. 4). No difference was observed within saline group before and 30 days after the injections (data not shown).

During a time course of 53 days in a model of streptozotocin-induced type 1 diabetes mellitus, morphological changes appear in the rat sciatic nerve. Some abnormalities include myelin infoldings, derangement in myelin compaction, and also a reduction in unmyelinated fibers starting 30 days after diabetes induction. These alterations worsen within 60 days after STZ injection (Fig. 4). Regarding the myelin organization, after laser treatment, we observed an improvement in STZ animals after four sessions of treatment (in Fig. 4d).

In Fig. 5, we can observe the quantification of myelin sheath obtained from transmission electron microscopy (8 animals per group). Note that the group of diabetic rats after laser

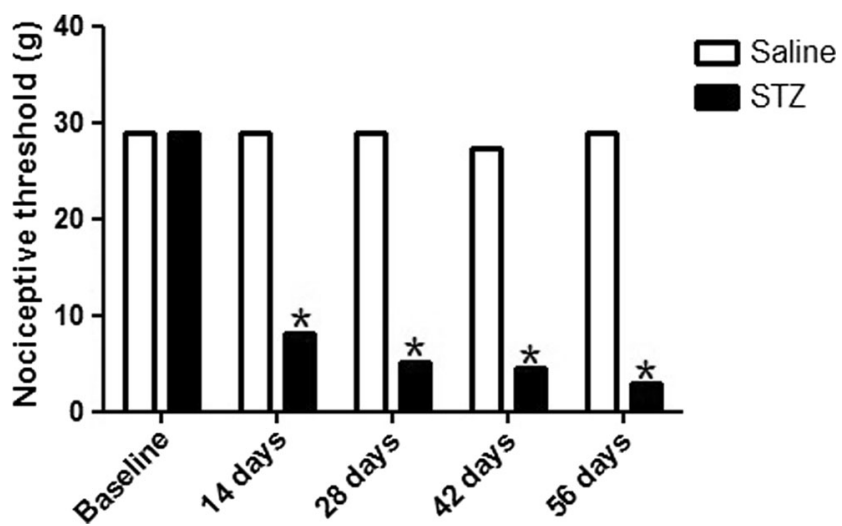

Fig. 2 Allodynia response in rats with streptozotocin. Nociceptive threshold measured by Von Frey hair (expressed in grams) was determined in saline (used as control group) and streptozotocin (STZ) groups, before (baseline) and on days 14, 28, 42, and on 56 days after both injections (saline and STZ). Results represent the mean $\mathrm{SEM} \pm 8-10$ animals per group. $* P<0.0001$ compared with saline 


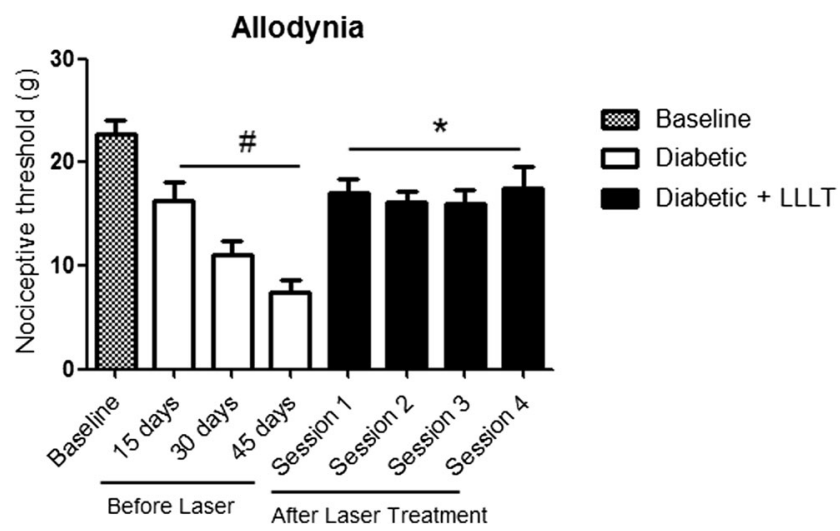

Fig. 3 Effect of laser therapy on nociceptive threshold measured by Von Frey hair (expressed in grams). Allodynic test was measured before (baseline) and after 15, 30, and 45 days after STZ injections. First session of laser therapy was applied after 45 days from STZ and measured every 2 days (STZ + LLLT). Results represent the mean $\mathrm{SEM} \pm 8$ animals per group $* P<0.05$ compared with $\mathrm{STZ}$ animals; ${ }^{\#} P<0.05$ compared with baseline

treatment does not show statistical differences in the thickness of myelinated nerve fibers when compared with saline group, used as control. Still, there were no statistical differences between naive and saline group (data not shown).

\section{Discussion}

High levels of glucose in the blood (hyperglycemia) induce abnormal structural changes in peripheral nerve fibers both myelinated and unmyelinated, which is a common characteristic of diabetic peripheral neuropathy. In association with other symptoms, chronic pain is the most prevalent symptom of those who suffer from diabetes, leading to a disabling life and affecting every aspect of a patient's life.

Here, we demonstrated that diabetic animals induced by a single dose of streptozotocin showed a decrease in their nociceptive thresholds. According to the results obtained in the present study, type 1 diabetes mellitus induced by streptozotocin has significantly effects on developing allodynia.
It was demonstrated that after four sessions of low level laser therapy, there is an increase in pain sensitivity. This effect was shown from the first session onwards, suggesting that low-level laser therapy could be a useful tool for patients with severe pain.

As mentioned in the "Introduction," the use of low-level laser therapy was first described in Europe and Russia in the 1960s [11], but its analgesic mechanisms are not yet well understood. In addition, previous studies have shown relevant data involving laser therapy and pain. Bingol et al. showed the effectiveness of low-level laser in patients with shoulder pain [25]. In a double blind study, Venancio et al. were able to show an improvement in patients with temporomandibular joint pain and also in mandibular dysfunction [26]. Previous studies from our group have also demonstrated the beneficial effects of laser therapy in animals with trigeminal nerve injury [23].

Moreover, the streptozotocin-induced type 1 diabetes mellitus model is able to cause significant nerve damage in myelinated as well as unmyelinated fibers. These findings are in accordance with previous data published about streptozotocin-induced diabetes mellitus and its chronic complications [27, 28].

In order to further evaluate the progression of desmielinization and other possible alterations after sptreptozotocin-induced type 1 diabetes mellitus, we designed a temporal analysis of sciatic nerve by eletron transmission microscopy. The sciatic nerve of diabetic rats was collected after 30 and 60 days of the disease for morphometric analysis of myelin sheath. Morphological changes in myelin sheath and fiber loss were clearly observed from the thirtieth day that worsened in the sixtieth after streptozotocin administration.

So according to these results, we decided to add a group of animals treated with low-level laser beginning 45 days after streptozotocin intraperitoneal administration. The choice of this timepoint was based on the behavioral tests where we noted that 45 days after streptozotocin administration there was a great decrease in pain sensitivity. At the end of the treatment, an improvement in myelin sheath structural organization as well as the reduction of fiber loss was observed when compared with

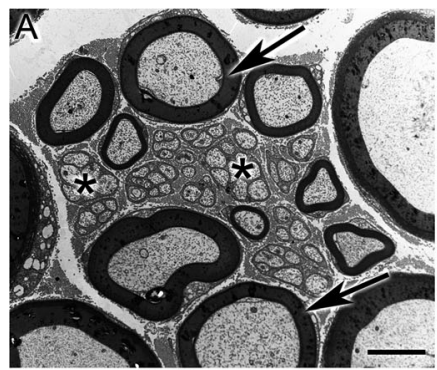

Saline

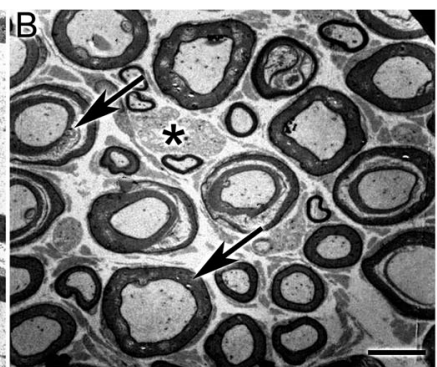

STZ 30 days

Fig. 4 Electron microscopic transverse section of the sciatic nerve. Myelinated nerve fibers are in normal morphology and structure in saline a control group ( 30 days). The photographs showed structural abnormality in myelin sheath in streptozotocin diabetic groups (b STZ

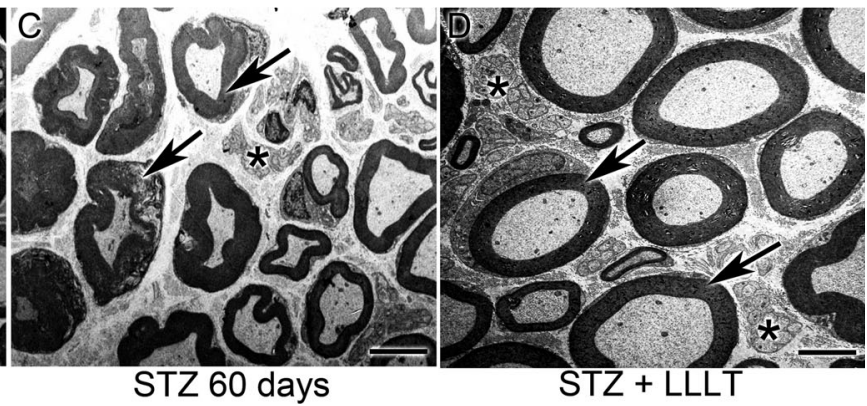

30 days, $\mathbf{c}$ STZ 60 days). d fibers from sciatic nerve after the last session of laser treatment (STZ + LLLT). Myelin Sheath (arrow), Schwann cell nucleus (arrow head), unmyelinated fibers (asterisk). Scale Bar $10 \mu \mathrm{m}$ 


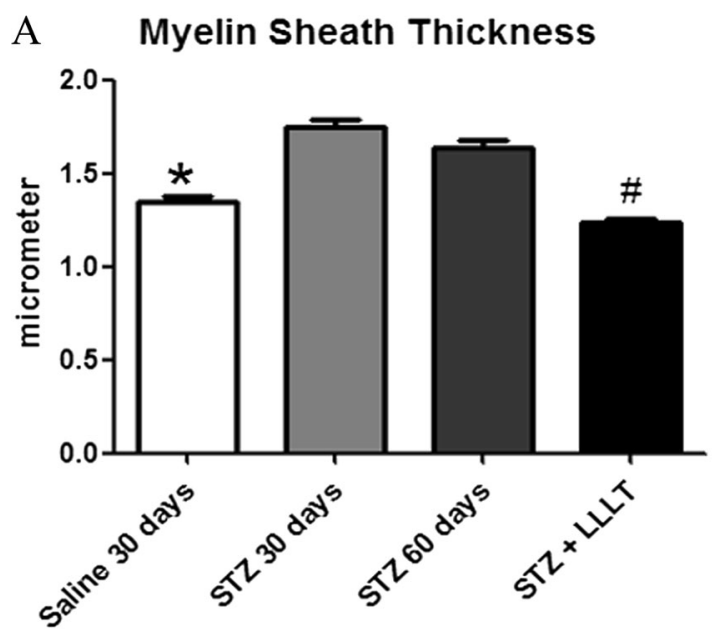

B

\begin{tabular}{lcccc}
\hline & $\begin{array}{c}\text { Saline } 30 \\
\text { days }\end{array}$ & $\begin{array}{c}\text { STZ30 } \\
\text { days }\end{array}$ & $\begin{array}{c}\text { STZ60 } \\
\text { days }\end{array}$ & STZ+ LLLT \\
\hline Myelin sheath & $1.37 \pm 0.24^{*}$ & $1.75 \pm 0.41$ & $1.64+0.30$ & $1.24+0.16^{2}$
\end{tabular}

Thickness

Fig. 5 Morphometric analysis of myelin sheath thickness of sciatic nerve. a the quantification of the images from myelin sheath. $\mathbf{b}$ the mean of the same animals during the days. Results represent the mean $\mathrm{SEM} \pm 8$ animals per group. $* p \leq 0.01$ indicates a significant difference between STZ 30 and STZ 60 days and saline; ${ }^{*} p \leq 0.05$ indicates a significant difference between the STZ + LLLT and diabetic animals (STZ 30 and STZ 60 days)

untreated groups. It is worth mentioning that despite the importance of the g-ratio for assessing axonal myelination, firstly described by Rushton [29], in this first approach, we intended to make some initial observations of low-level laser therapy in a 904-nm wavelength on nociceptive thresholds (allodynia) and if this treatment would probably have beneficial effects on sciatic nerve fibers in diabetic rats. In addition, this study is not intended to evaluate nerve conduction.

\section{Conclusion}

These findings may contribute to previous studies that showed other event may be in relation with pain symptoms in diabetes mellitus not only the degeneration itself.

We can attribute methylglyoxal, an alpha-carbonyl, with a possible role in the activation of sodium channels in peripheral nerves, contributing to the pain sensitivity observed in diabetic rats [30]. It should be mentioned that more research needs to be done to improve our knowledge about how hyperglycemia induces peripheral nerve damage and chronic pain.

Despite the lack of biochemical and molecular studies to confirm our results, we can suggest and ensure that low-level laser therapy is a useful tool for treating pain in diabetic animals. Moreover, this same therapy was able to prevent morphological changes and loss of peripheral unmyelinated and myelinated fibers.

Acknowledgments FAPESP 2014/24533-0.

Authors' contributions All authors made substantial contributions to the following tasks of research: initial conception (Rocha, I.R.C; Martins, D.O. Chacur, M.); design (Rocha, I.R.C; Martins D.O., Chacur M); provision of resources (Chacur M); collection of data (Rocha, I.R.C; Rosa, S. Ciena A.P); analysis and interpretation of data (Rocha, I.R.C; Chacur M.); writing the first draft of the paper or important intellectual content (Rocha, I.R.C; Martins D.O., Chacur M.); and revision of the paper (Rocha, I.R.C; Martins D.O., Chacur M).

\section{Compliance with ethical standards}

Ethics approval and consent to participate All procedures were approved by the Institutional Animal Care Committee of the University of São Paulo (protocol number 123/2015) and performed in accordance with the guidelines for the ethical use of conscious animals in pain study published by the International Association for the Study of Pain.

Consent for publication Not applicable.

Availability of data and materials Not applicable.

Competing interests The authors declare that they have no competing interests.

Funding FAPESP 2014/24533-0.

\section{References}

1. Federation ID (2013) Diabetes Atlas; Available from: http://www. idf.org/diabetesatlas

2. Javed S, Petropoulos IN, Alam U, Malik RA (2015) Treatment of painful diabetic neuropathy. Ther Adv Chronic Dis 6:15-28

3. Jin HY, Lee KA, Song SK, Liu WJ, Choi JH, Song CH, Baek HS, Park TS (2012) Sulodexide prevents peripheral nerve damage in streptozotocin induced diabetic rats. Eur J Pharmacol 674:217-26

4. Yagihashi S, Mizukami H, Sugimoto K (2011) Mechanism of diabetic neuropathy: where are we now and where to go? J Diabetes Investig 2:18-32

5. Cermenati G, Abbiati F, Cermenati S, Brioschi E, Volonterio A, Cavaletti G, Saez E, De Fabiani E, Crestani M, Garcia-Segura LM, Melcangi RC, Caruso D, Mitro N (2012) Diabetes-induced myelin abnormalities are associated with an altered lipid pattern: protective effects of LXR activation. J Lipid Res 53:300-10

6. Azcoitia I, Leonelli E, Magnaghi V, Veiga S, Garcia-Segura LM, Melcangi RC (2003) Progesterone and its derivatives dihydroprogesterone and tetrahydroprogesterone reduce myelin fiber morphological abnormalities and myelin fiber loss in the sciatic nerve of aged rats. Neurobiol Aging 24:853-60

7. Veiga S, Leonelli E, Beelke M, Garcia-Segura LM, Melcangi RC (2006) Neuroactive steroids prevent peripheral myelin alterations induced by diabetes. Neurosci Lett 402:150-3

8. Services USDoHaH (2009) Diabetic neuropathies: the nerve damage of diabetes

9. Javed S, Alam U, Malik RA (2015) Treating diabetic neuropathy: present strategies and emerging solutions. Rev Diabet Stud 12:6383 
10. Kaur S, Pandhi P, Dutta P (2011) Painful diabetic neuropathy: an update. Ann Neurosci 18:168-75

11. Ribas ES, Paiva WS, Pinto NC, Yeng LT, Okada M, Fonoff ET, Chavantes MC, Teixeira MJ (2012) Use of low intensity laser treatment in neuropathic pain refractory to clinical treatment in amputation stumps. Int J Gen Med 5:739-42

12. Kingsley JD, Demchak T, Mathis R (2014) Low-level laser therapy as a treatment for chronic pain. Front Physiol 5:306

13. Passarella S (1989) He-Ne laser irradiation of isolated mitochondria. J Photochem Photobiol B 3:642-3

14. Yamamoto H OA IN, Kinoshita S (1988) Antinociceptive effects of laser irradiation of Hoku point in rats. Pain Clin

15. L-AL AC (199979-87) Beneficial effects of laser therapy in the early stages of rheumatoid arthritis onset. Laser Ther

16. Zimmermann M (1983) Ethical guidelines for investigations of experimental pain in conscious animals. Pain 16:109-10

17. Cheng Y, Kang H, Shen J, Hao H, Liu J, Guo Y, Mu Y, Han W (2015) Beta-cell regeneration from vimentin+/MafB+ cells after STZ-induced extreme beta-cell ablation. Sci Rep 5:11703

18. Araiza-Saldana CI, Pedraza-Priego EF, Torres-Lopez JE, RochaGonzalez HI, Castaneda-Corral G, Hong-Chong E, GranadosSoto V (2015) Fosinopril prevents the development of tactile allodynia in a streptozotocin-induced diabetic rat model. Drug Dev Res 76:442-9

19. Ciena AP, de Almeida SR, Dias FJ, Bolina Cde S, Issa JP, Iyomasa MM, Ogawa K, Watanabe IS (2012) Fine structure of myotendinous junction between the anterior belly of the digastric muscle and intermediate tendon in adults rats. Micron 43:258-62

20. Watanabe I, Yamada E (1983) The fine structure of lamellated nerve endings found in the rat gingiva. Arch Histol Jpn 46:173-82
21. da Silva JT, Santos FM, Giardini AC, Martins Dde O, de Oliveira ME, Ciena AP, Gutierrez VP, Watanabe IS, Britto LR, Chacur M (2015) Neural mobilization promotes nerve regeneration by nerve growth factor and myelin protein zero increased after sciatic nerve injury. Growth Factors 33:8-13

22. Chaplan SR, Bach FW, Pogrel JW, Chung JM, Yaksh TL (1994) Quantitative assessment of tactile allodynia in the rat paw. J Neurosci Methods 53:55-63

23. de Oliveira MD, Martinez dos Santos F, Evany de Oliveira M, de Britto LR, Benedito Dias Lemos J, Chacur M (2013) Laser therapy and pain-related behavior after injury of the inferior alveolar nerve: possible involvement of neurotrophins. J Neurotrauma 30:480-6

24. Snedecor GW RS, FJ Rohlf (1946) Statistical methods biometry. Freeman \& Co

25. Bingol U, Altan L, Yurtkuran M (2005) Low-power laser treatment for shoulder pain. Photomed Laser Surg 23:459-64

26. Venancio Rde A, Camparis CM, Lizarelli Rde F (2005) Low intensity laser therapy in the treatment of temporomandibular disorders: a double-blind study. J Oral Rehabil 32:800-7

27. Hanani M, Blum E, Liu S, Peng L, Liang S (2014) Satellite glial cells in dorsal root ganglia are activated in streptozotocin-treated rodents. J Cell Mol Med 18:2367-71

28. Hoybergs YM, Meert TF (2007) The effect of low-dose insulin on mechanical sensitivity and allodynia in type I diabetes neuropathy. Neurosci Lett 417:149-54

29. Rushton WA (1951) A theory of the effects of fibre size in medullated nerve. J Physiol 115:101-22

30. Huang Q, Chen Y, Gong N, Wang YX (2016) Methylglyoxal mediates streptozotocin-induced diabetic neuropathic pain via activation of the peripheral TRPA1 and Nav1.8 channels. Metabolism 65: 463-74 